\title{
Use of Ducting Theory in an Observed Case of Gravity Waves
}

\author{
S. MONSERRAT \\ Departament de Física, Universitat Illes Balears and Instituto Mediterráneo de Estudios Avanzados, \\ IMEDEA (CSIC-UIB), Palma de Mallorca, Spain
}

\author{
A. J. THORPE \\ Department of Meteorology, Universiry of Reading, Reading, United Kingdom
}

(Manuscript received 6 September 1994, in final form 18 December 1995)

\begin{abstract}
This paper attempts to explain the properties of a gravity wave event observed on Mallorca (Balearic islands) using an array of microbarographs. The waves propagated coherently for a relatively long distance with a speed of about $29 \mathrm{~m} \mathrm{~s}^{-1}$ and were nondispersive. In the absence of a continuous forcing, long-lived atmospheric gravity waves can only exist if some mechanism prevents the vertical leakage of energy through wave propagation, trapping the gravity wave in a duct layer near the surface. Lindzen and Tung showed the necessary conditions for trapping and discussed the properties of neutral modes for constant stability and wind in the duct. The role of shear in the wind profile near the ground is examined here by first finding three distinct neutral mode types using a matrix eigenvalue method. Applying the wave theory given by Booker and Bretherton, those neutral modes in the sheared duct that have critical levels within the stable duct will be mostly absorbed at their critical level. Therefore, they will be negligible in surface pressure measurements. Another mode type has no critical level and propagates energy away from the duct. On the other hand, neutral modes with a critical level above the top of the duct will be reflected and so will constitute the main signal in the surface observations. This appears to explain observations in Mallorca and shows the significant role of wind shear in selecting the phase speed of the ducted waves.
\end{abstract}

\section{Introduction}

In recent years, numerous articles have considered the source mechanisms and propagation of gravity waves in the lower atmosphere. Different studies suggest gravity waves can be generated by either flow over mountains, convection, Kelvin-Helmholtz instability, or geostrophic adjustment (e.g., Gossard and Hooke 1975; Einaudi 1980). Nonlinear wave-wave interaction, suggested by some authors (Fritts 1982, 1984), can only modify the wave spectrum and cannot be considered as a source of wave activity itself.

A forcing mechanism is, of course, necessary to trigger gravity waves, but, on some occasions, remarkably large amplitude atmospheric disturbances have been recorded in regions where a continuous forcing is not observed or is far away from the suggested source region. On these occasions, whatever the energy source for these waves may be, long-lived gravity waves can only exist if some mechanism is able to trap the wave energy near the surface throughout the wave propaga-

Corresponding author address: Dr. Sebastian Monserrat, Department of Physics, University of the Balearic Islands, E-07071 Palma de Mallorca, Spain.

E-mail: dfssmt4@ps.uib.es tion. Otherwise, they would lose their energy through vertical propagation, normally before traveling a complete wavelength in the horizontal.

In summer 1990, some gravity wave events were detected on the Balearic islands (western Mediterranean) by measuring surface pressure with a triangular array of microbarographs (Monserrat and Thorpe 1992). The waves were detected far away from any significant orography that might be considered responsible for the wave generation, and no convection was normally reported. On the other hand, the gravity waves were observed to be long-lived, traveling long distances without significant loss of coherence. The presence of some mechanism trapping the energy near the surface must have occurred in order to explain the good correlations observed.

Lindzen and Tung (1976), hereafter referred to as LT, provided one of the most complete works on gravity wave trapping. They show the necessary conditions for the existence of an efficient wave duct from which little wave energy leaks. The duct must be a stable layer adjacent to the surface, deep enough to maintain at least a quarter of the vertical wavelength of the ducted mode, and capped by a good reflector. An unstable layer, where the Richardson number is less than 0.25 , containing a critical level optimizes the reflection. This lat- 
ter layer above the wave duct would reflect the wave energy and would allow the wave propagation for several waves cycles without significant loss of energy.

Vertical profiles of the atmosphere observed on the Balearic islands at the time of the gravity wave events exhibit a level at about $4 \mathrm{~km}$ where the wave-trapping condition given by LT is satisfied. This indicates the possible presence of a wave duct trapping the energy at lower levels. The LT results are based on a model with constant wind and Brunt-Väisälä frequency within the duct. Radiosonde ascents made from the Balearic islands clearly show that the wind speed increases with height in the stable layer near the ground. Here the role of these variable atmospheric profiles on free modes is described. This is one way to show the properties of the waves existing in the duct. We use the trapping conditions given by LT to suggest which of these modes will be trapped and be observed at the ground. This constitutes the main aim of this paper.

In general, we assume wave solutions of the form $W$ $=W(z) e^{i k(x-c t)}$, where $W$ is a scaled vertical velocity $\left(W=\rho_{0}^{1 / 2} w\right.$, where $\rho_{0}$ is the atmosphere's mean density and $w$ is the vertical component of the perturbation wind speed), $k$ the horizontal wavenumber, the $x$ axis is in the direction of the wave phase propagation, and $c$ the phase speed. The perturbation two-dimensional governing gravity wave equations can then be reduced to a single equation for $W$ with the form

$$
\left(\frac{d^{2}}{d z^{2}}+\lambda^{2}(z)\right) W(z)=0
$$

known as the Taylor-Goldstein equation (Goldstein 1931; Taylor 1931).

In this paper, we solve the Taylor-Goldstein equation using the matrix method as described in the appendix. In particular, we study the influence of the wind shear on neutral free modes. The results are used to explain the properties of the gravity waves observed on the Balearic Islands in summer 1990. In section 2 the free modes are obtained assuming first an idealized constant wind and afterwards including a constant wind shear in the duct. Then in section 3 an interpretation of these modes in terms of wave reflection and absorption is given. In section 4 these results are discussed in connection with an episode of gravity waves in summer 1990 in the Balearic Islands. Evidence that the observed gravity waves were neutral modes trapped in a duct is given by invoking the trapping conditions suggested by LT. The results obtained with this model are in very good agreement with the observations giving an explanation for the nondispersive nature of the waves. Finally, in section 5 a summary of the main results and the conclusions are presented.

\section{Free modes in stable duct profiles}

In this section, some idealized profiles are used to determine the characteristics of the neutral and growing modes in a layer near the ground. Related research includes Lindzen and Rosenthal (1976, LR hereafter), Rosenthal and Lindzen (1983), and Monserrat and Ramis (1990). Instead of attempting to describe the complete parameter space of the dependence of the growth rate and phase speed as a function of the determining nondimensional parameters describing the profile, we set typical dimensional values suggested from the observations from Mallorca. Here we concentrate on the horizontal wavenumber dependence of the growth rates and phase speeds, with special attention to the neutral modes and the dispersive nature of the waves. This will be compared with and without shear in the layer near the ground.

The matrix method, as described in the appendix, yields $M$ eigenfunctions ( $M$ being the number of vertical levels), many of which are spurious, regardless of how many modes the continuous profile can support. To take this into account, computations have been carried out for different resolutions to check if the modes found are physical or solely related to the selected number of vertical levels. The presence of the spurious modes has been shown to be more evident when computing growth rates of unstable modes for short wavelengths. It has been found that spurious modes are mixed with physical ones when insufficient vertical resolution is used. As very short wavelengths are not detected by the microbarograph array, we choose a vertical resolution sufficient to describe the observed waves. All the results shown have been obtained using the matrix method with 100 vertical levels and a domain of total height of $6000 \mathrm{~m}$, implying a resolution of $60 \mathrm{~m}$. A rigid lid is assumed as the upper-boundary condition. From a practical viewpoint, it is not possible to include a radiation condition at the upper boundary in the version of the matrix method we use. Any radiative condition implies the eigenfunction should behave as $\exp (i m z)$ above a given height, where $m$ is the vertical wavenumber depending on the actual value of the phase speed. This can be used in any "shooting method" based on a first guess for the phase speed. The final correct solution is found by an iterative process. In the method we use, the eigenvalue (phase speed) is not known a priori. The possibility of obtaining the solutions without implementing any first guess has some advantages, but it also implies that a radiation condition cannot be implemented. The use of a rigid lid at the top of the domain then requires justification, as it clearly implies strong reflection at that level. (In the atmosphere the tropopause acts as a partially reflective upper boundary, so one can question the realism of an entirely radiative upper boundary also.)

Our attention in this paper focuses only on waves that have a significant surface pressure signature. These are ducted waves that have, in any case, very small amplitudes in the upper levels. Therefore, the results of the matrix method for these modes are expected to be insensitive to the choice of the upper-boundary condi- 
tion. This is supported by the fact that when the top of the model is selected at a higher level or the number of levels is increased, the results for these modes are very similar. This is not true, however, for those modes found to have a significant response near the upper boundary. These modes can be considered as spurious since they depend on the position of the unrealistic upper boundary. We refer to these modes in the text, although it should be kept clearly in mind that they are related to the presence and position of the upper rigid lid. This is similar to the analysis presented by Lindzen et al. (1968), in which the behavior of several models with and without the presence of a top are compared. Those oscillations propagating in the vertical are expected to be strongly modified by the presence of the upper rigid lid, although those evanescent in the vertical should be insensitive to the upper boundary condition. The modes we are interested in propagate in the vertical from the surface to a given height below the upper boundary and are evanescent from this height upward.

\section{a. Constant wind in the duct}

We apply the method to the case of a constant wind in a layer with $\mathrm{Ri}=\infty$ (the lower layer), capped by a dynamically unstable (intermediate) layer where $\mathrm{Ri}$ $<0.25$ and above which the wind returns to being constant with height (the upper layer) (Fig. 1a). The Richardson number, $\mathrm{Ri}$, is defined in each layer as

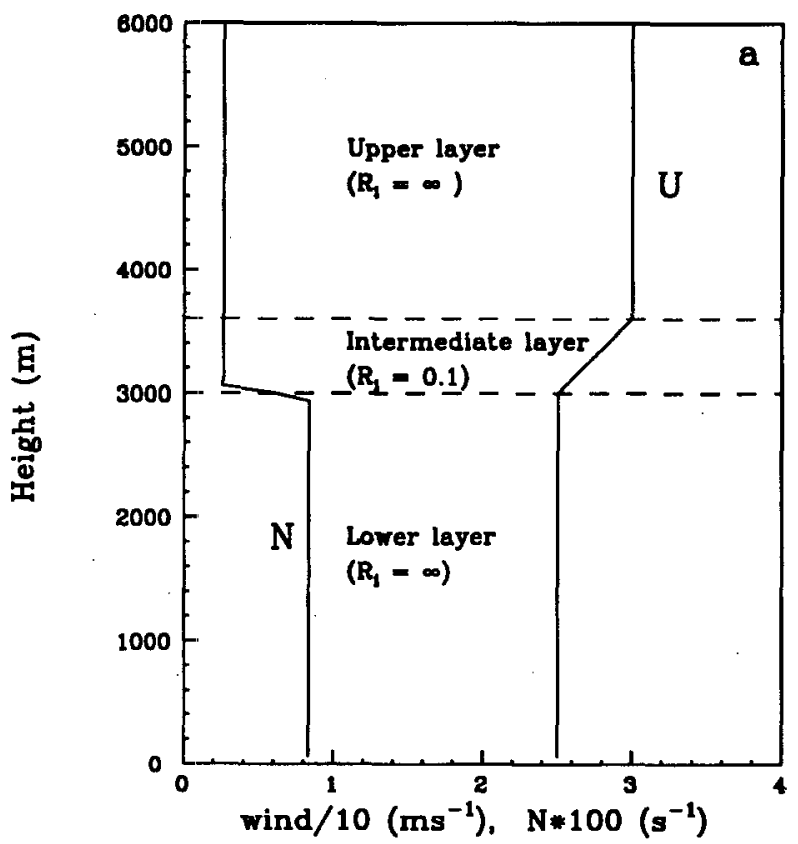

$$
\mathrm{Ri}=\frac{N^{2}}{(d U / d z)^{2}},
$$

where $U$ is the wind speed, $z$ is the height, and $N$ is the Brunt-Väisälä frequency given by

$$
N^{2}=-\frac{g}{\rho_{0}} \frac{d \rho_{0}}{d z}-\frac{g^{2}}{C_{s}^{2}}
$$

where $g$ is the gravitational acceleration and $C_{s}$ is the speed of sound (assumed constant).

A further case involves using the same profiles except that the wind in the lower layer has constant shear (Fig. 1b). This will allow the simplest description of the modification of the results due to shear in the duct and is presented in section $2 b$.

The profile in Fig. 1a is the same as that used by Rosenthal and Lindzen (1983) to study instabilities in stratified fluids with one critical level. It is also similar to the profile used by LR, although in that paper the depth of the intermediate layer is set to zero and the vertical velocity is not forced to be zero at the upper boundary in either of the two studies. It also can be compared with that used in Monserrat and Ramis (1990), but here no discontinuity in the wind profile is considered, and the Brunt-Väisälä frequency is not set to zero in the intermediate sheared layer.

We first apply the method to find the unstable modes that the profile in Fig. 1a can support. This analysis is of interest as it allows a verification of the matrix method by comparing its modes with those obtained by

FIG. 1. Wind $(U)$ and Brunt-Väisälä frequency $(N)$ for the profiles used in section two, (a) with constant wind in the lower layer and (b) with constant wind shear in the lower layer. The name of the different layers and the value of the Richardson number in each layer are also indicated. 
previously mentioned authors who considered instabilities. Also, it will be important in judging whether the observed gravity waves are more likely to be unstable or neutral.

The wind $(U)$ in this profile is symmetric about a mean value of $U_{0}=27.5 \mathrm{~m} \mathrm{~s}^{-1}$ in the middle of the intermediate layer. To describe the modes, it is useful to refer the results to this value. A number of unstable modes selected by their phase speeds are found. The phase speeds of the unstable modes range between 25 and $30 \mathrm{~m} \mathrm{~s}^{-1}$, the minimum and maximum wind speed for this profile. This is consistent with the Miles-Howard semicircle theorem (Howard 1961), which restricts the phase speed relative to the mean wind $\left(c-U_{0}\right)$ for unstable solutions to a semicircle of radius $\delta U$, where $\delta U=U_{1}-U_{0}$ and $U_{1}$ is the wind speed in the top layer. We can divide the unstable modes into two types: one with phase speeds greater than $U_{0}$, and the other with phase speeds less than $U_{0}$. For each mode with phase speed $c_{1}<U_{0}$, another mode is found with phase speed $c_{2}=2 U_{0}-c_{1}$ and the same growth rate. Only the first type was found by LR. The second type appears as a consequence of having a rigid upper boundary. In Fig. 2, the growth rate and the phase speed for the two most unstable pairs of modes are shown.

The neutral modes can be divided into two basic categories: modes with a significant pressure response at the surface, and modes located at higher levels without any such response near the ground. To compare the different modes, we give the maximum vertical velocity an arbitrary magnitude of unity.

We believe it is significant that the results for neutral modes with significant response at the surface have a resemblance to those from LR, shown in Fig. 3. These modes are found here in spite of the use of the rigid upper boundary. This gives confidence that the upper rigid lid does not disturb those modes that have, by their nature, little response at upper levels. These are the only modes found by LR. The reason why other modes are found here should be attributed to the presence of the upper boundary and can be considered as spurious, in the sense that their structure depends on the actual position of the upper lid. As we are particularly interested in the neutral modes with significant response near the surface, there will be no further discussion of the modes that are restricted to upper levels.

Lindzen and Rosenthal (1976) analytically found an infinite number of neutral modes $(m=1,2,3, \cdots)$, each having $m / 2$ vertical wavelengths in the lower layer. For each mode number, two types of solutions were found, one with $-U<c<0$ and the other with $c<-U$, when the wind in the lower and the upper layer was assumed to be $-U$ and $U$, respectively. A similar result is reproduced here: two modes with about
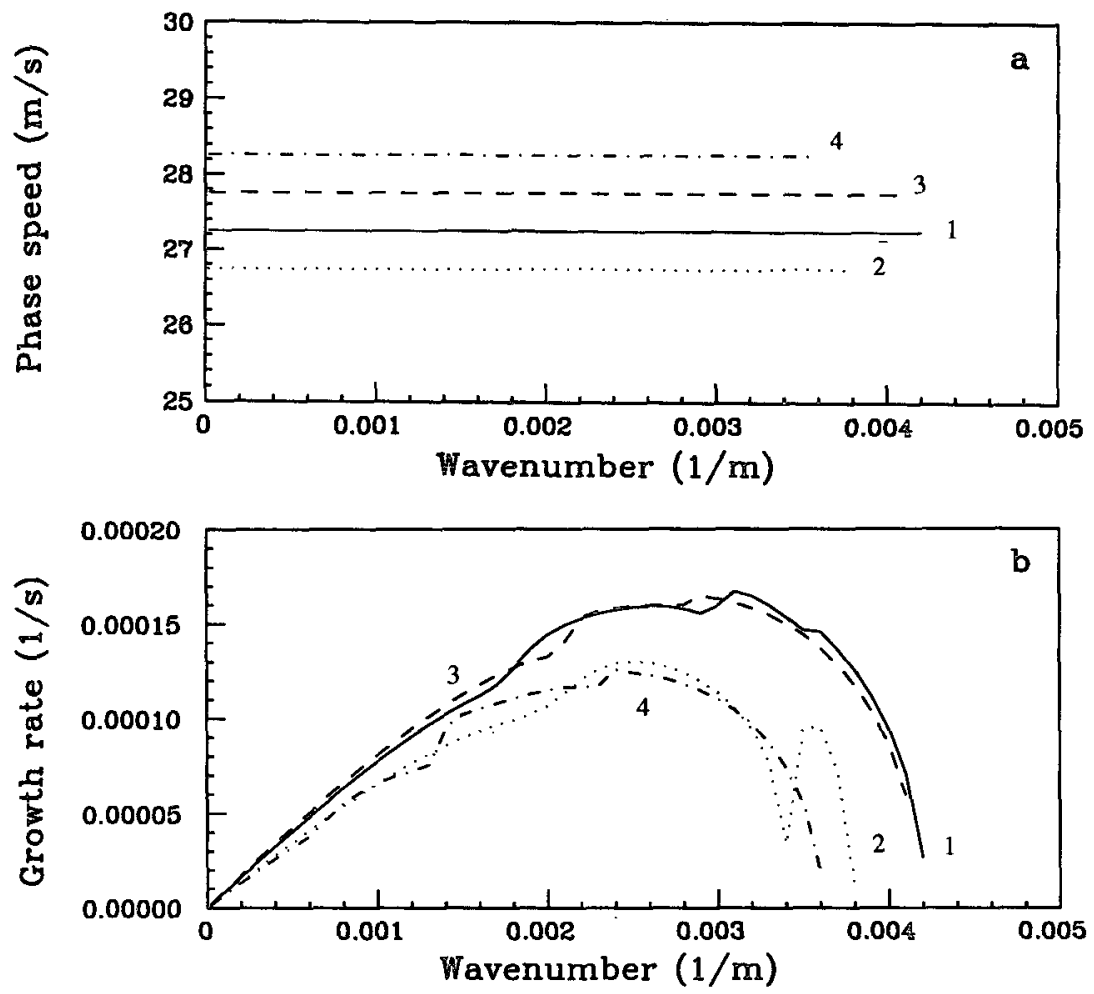

FIG. 2. (a) Phase speed and (b) growth rate plotted against wavenumber of the four most unstable modes for the profiles in Fig. 1a. The growth rate for modes 1 and 3 and for modes 2 and 4 are almost identical. 

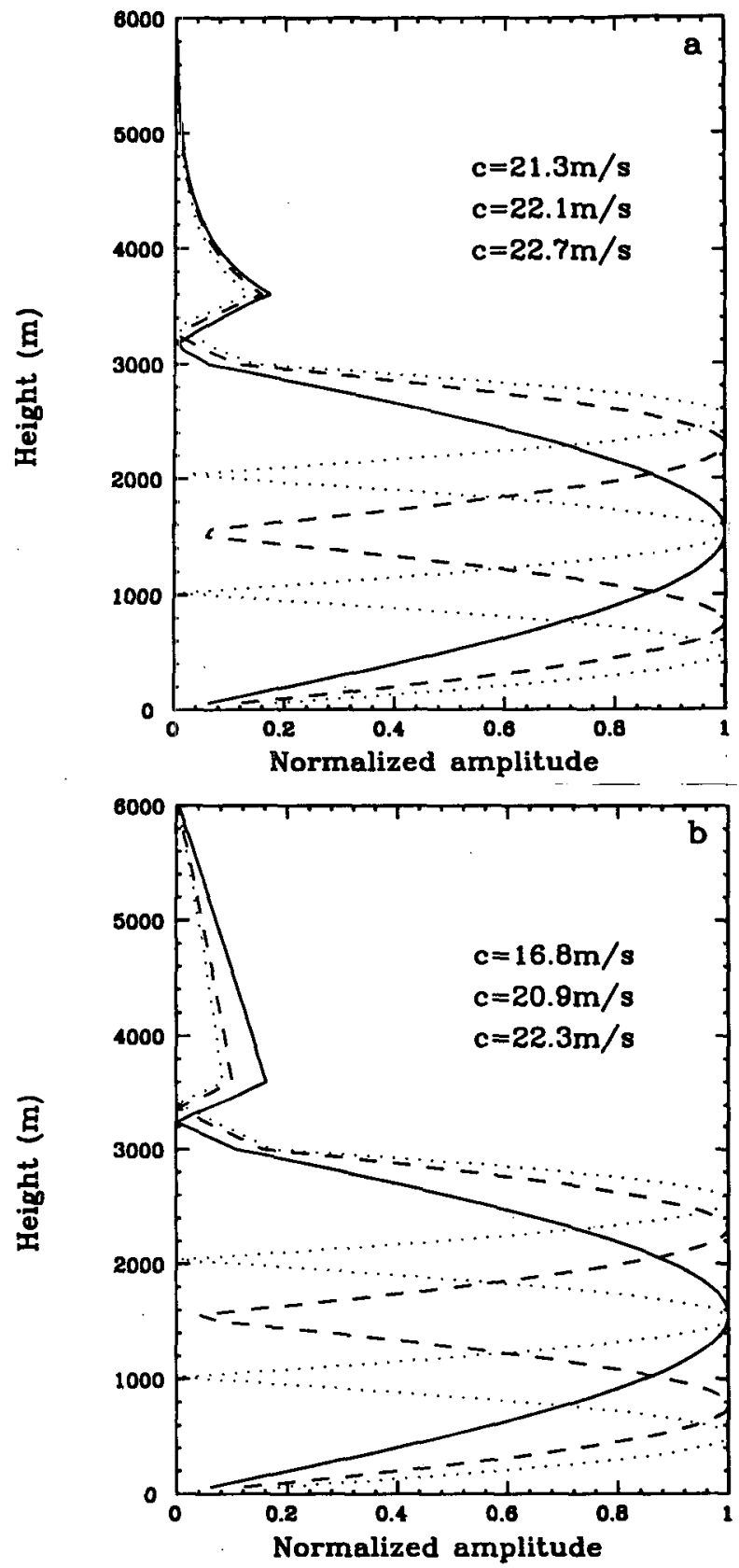

FIG. 3. The modulus of the normalized vertical velocity for three neutral modes, supported by the profiles given in Fig. 1a. The wavenumber is $k=2 \times 10^{-3} \mathrm{~m}^{-1}$ in (a) and $k=5 \times 10^{-5} \mathrm{~m}^{-1}$ in (b). The phase speeds of the modes are indicated in the figure.

half of a vertical wavelength in the lower layer $(m=1$ in LR), one with $c<25 \mathrm{~m} \mathrm{~s}^{-1}$ and the other with $c$ $>25 \mathrm{~m} \mathrm{~s}^{-1}$, together with a number of other higherorder modes are found. The phase speeds of the " $m$ $=1$ modes" are the smallest and the largest phase speeds the profiles can support, and this range of allowed phase speeds decreases when the wavenumber increases. In Fig. 3a, the normalized vertical velocity of three of these neutral modes for $k=2 \times 10^{-3} \mathrm{~m}^{-1}$ $(\lambda=3 \mathrm{~km})$ are plotted versus height. The phase speeds of the two $m=1$ modes are for this case $c_{\min }=21.3$ $\mathrm{m} \mathrm{s}^{-1}$ and $c_{\max }=28.7 \mathrm{~m} \mathrm{~s}^{-1}$. Only the mode with speed $c_{\min }=21.3 \mathrm{~m} \mathrm{~s}^{-1}$ is shown in Fig. 3a. In Fig. 3b, the normalized vertical velocities for these modes, but now for $k=5 \times 10^{-5} \mathrm{~m}^{-1}(\lambda=126 \mathrm{~km})$, are shown. The vertical structure of the modes is similar, but the range of allowed phase speeds is greater than for the case in Fig. 3a. Now, $c_{\min }=16.8 \mathrm{~m} \mathrm{~s}^{-1}$ and $c_{\max }=32.4$ $\mathrm{m} \mathrm{s}^{-1}$.

Another result, already pointed out by $L R$, is that higher-order modes are nondispersive, and also, for large enough $k$, the phase speeds of all the modes are essentially independent of $k$. The value of the wavenumber at which the phase speed starts to noticeably change with $k$ depends on the parameters in the model (i.e., wind and Brunt-Väisälä frequency). From a practical point of view, the observed waves would appear to be essentially nondispersive if one of the higher order modes dominates or if the observable wavelengths are small enough to be in the nondispersive region even when no mode is dominant.

No other comments about how these modes are modified by other values of wind and Brunt-Väisälä frequency are made here. On the other hand, one of the aims of this work is to show how the presence of wind shear in the lower layer modifies these results.

\section{b. Constant wind shear in the duct}

First, we look at the unstable modes for the profiles in Fig. $1 \mathrm{~b}$ and then compare them with the results found with constant wind in the lower layer. The results are similar to those found without shear in the lower layer, although now the growth rates are larger and the most unstable wavelengths are shorter. Also, the phase speeds of the unstable modes range between 0 and 30 $\mathrm{m} \mathrm{s}^{-1}$, although small phase speeds are associated with small growth rates.

Five unstable modes for the profile shown in Fig. 1b are shown in Fig. 4. They include the most unstable mode the profile can support with in the range of wavenumbers shown $\left(\lambda=722 \mathrm{~m}, c=25.37 \mathrm{~m} \mathrm{~s}^{-1}\right)$. Modes with larger growth rates may exist for larger wavenumbers (Kelvin-Helmholtz instabilities). However, better vertical resolution is needed to describe these shorter-wavelength modes. Kelvin-Helmholtz modes with very short vertical and horizontal scales cannot be resolved properly with a given vertical resolution. Spurious modes for these short horizontal wavelengths are mixed with physical ones, and the results for growth rates are corrupted. This means that the matrix method needs a good vertical resolution to properly find shortwavelength modes. As short wavelengths cannot be detected by the microbarograph observations described here, they are not discussed any further. 

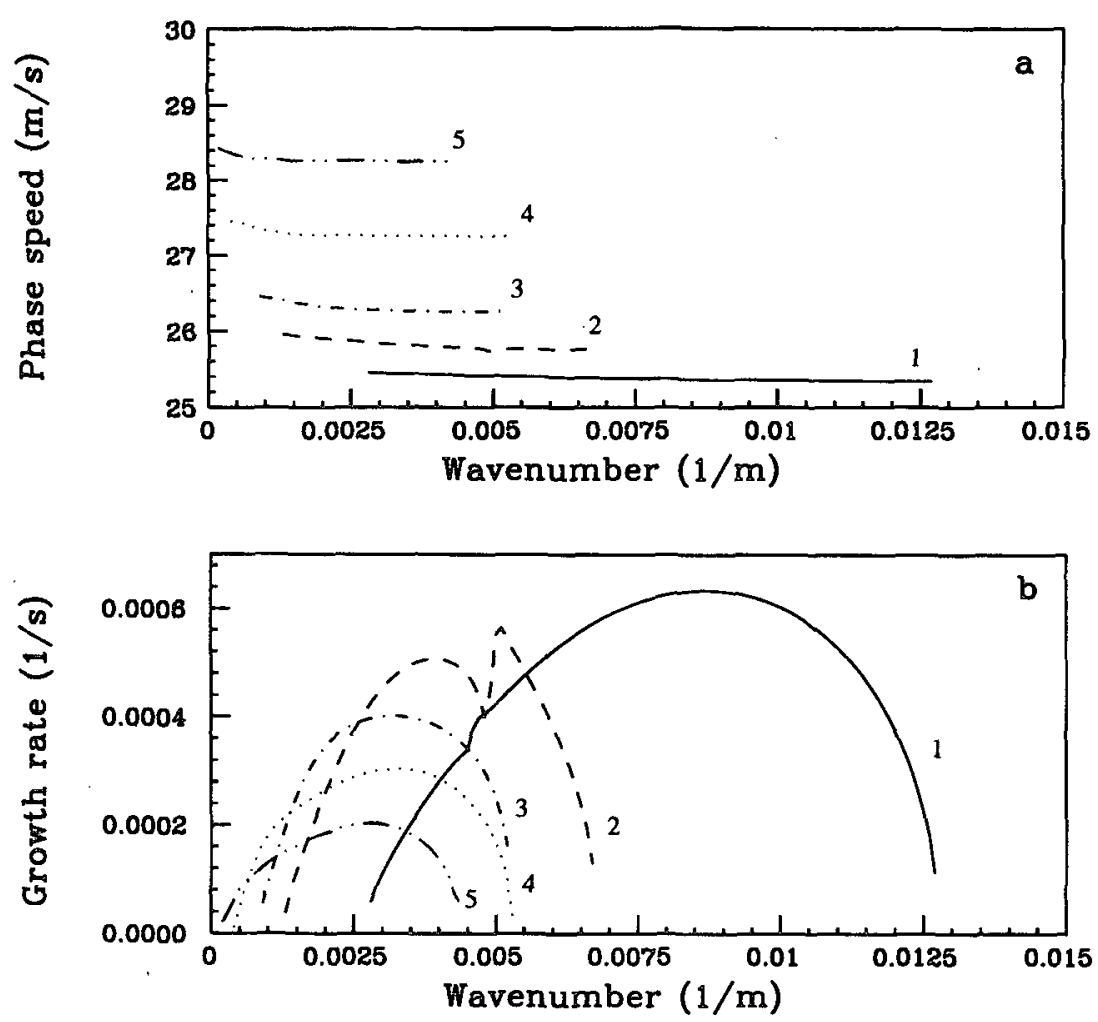

Fig. 4. (a) Phase speed and (b) growth rate plotted against wavenumber of five unstable modes for the profiles in Fig. 1b. The growth rate for mode 2 has a different behavior, probably because it is formed by two different overlapping modes.

On the other hand, the matrix method for the sheared profile finds a number of neutral modes that have a clearly different behavior than that found with constant wind in the lower layer. We can divide these modes into two different types: 1) type A, located at higher levels without a significant response near the ground and 2 ) type $B$, with a significant response near the ground.

Examples of the vertical structure of these two modes for $k=8 \times 10^{-4} \mathrm{~m}^{-1}(\lambda=7.85 \mathrm{~km})$ are given in Fig. 5. A difference found in the modes when the lower layer had a constant wind speed is that the small vertical wavelengths present in the model with a constant wind speed in the lower layer, as shown in Fig. 3 , are no longer present in the model with wind shear in the lower layer. Instead, their response is restricted in some narrow layer near the ground, as shown for example in Fig. 5b. Type A modes are not of interest here as they do not contribute to the observed surface pressure signature, are strongly dependent on the position of the upper lid, and are likely spurious artifacts of the presence of the rigid upper boundary in the model, which would not be present with a more realistic radiative upper boundary condition and hence would not be present in the atmosphere. The focus of the rest of the paper will be on type B modes. Within type $B$, we can distinguish three subclasses depending on their phase speeds:

1) type B1, with phase speeds less than the wind speed at the top of the lower layer but greater than that at the ground;

2) type B2, with a phase speed equal to the wind speed in the intermediate layer;

3) type B3, with a phase speed greater than the maximum wind speed in the domain.

At this stage, it is important to discuss the precise mathematical nature of the matrix eigenvalues. The numerical method finds modes of a steplike wind profile and not the continuous wind shear profile. For type B1 and B2 modes, it is found that the numerical phase speeds lie between the mean wind values of each step, so that there is formally no critical-level behavior of the steplike profile. It is far from obvious whether increasing the vertical resolution in the numerical method will converge to the same solutions as could be found from a (hypothetical) analytical solution. The continuous profile will, by definition, exhibit critical-level behavior for these modes. Any high-resolution numerical calculation will not, however, produce such criticallevel behavior. However, these numerical modes are 
not mathematically incorrect. Actually, they are real modes of the steplike profile. They must, it seems, be regarded as being equivalent (but not identical) to a set of critical-level modes of the continuous profile. As such, they will, for the continuous shear profile, be subject to absorption at their critical level and, in accordance with theorem IX of Miles (1961), they will not exist as singular neutral modes.

\section{Interpretation of modes in terms of wave trapping}

It appears, from the observational evidence from the Mallorcan microbarographs, that the waves observed at the ground have a very small range of phase speeds. This suggests that only some of the numerically calculated modes are being observed at the surface. Here, we attempt to give a physical explanation of this in terms of wave absorption and reflection, as described by Booker and Bretherton (1967) and Lindzen and Tung (1976), respectively.

Lindzen and Tung (1976), analyzing the necessary conditions for the presence of ducted waves in the atmosphere, basically discussed the necessary properties of an effective reflecting layer able to maintain wave energy near the surface. They showed that the presence of a critical level, or at least a nearly critical level, either in or above an unstable layer capping a stable duct is optimal for reflecting the waves. If we assume that the reflecting layer is present, the ducted wave properties will be governed by the duct characteristics. Assuming simple atmospheric profiles for wind and Brunt-Väisälä frequency in the duct, some general comments can be made. Some of these have already been noted by LT.

In the Taylor-Goldstein equation [Eq. (1)], $\lambda^{2}(z)$ is normally written as

$$
\lambda^{2}(z)=l^{2}(z)-k^{2},
$$

where $l^{2}(z)$ is known as the Scorer parameter.

The vertical propagation of the gravity waves is basically controlled by $l^{2}(z)$, which can be approximated (neglecting compressible terms and the terms including the second derivative of $U$ ) by the expression

$$
l^{2}(z)=\frac{N^{2}}{(U-c)^{2}} .
$$

When the Scorer parameter is larger than $k^{2}$, the vertical wavenumber is real, representing oscillatory waves. On the other hand, when it is smaller than $k^{2}$, the solutions for $\lambda(z)$ are imaginary, the wave is evanescent, and its amplitude decays exponentially with distance away from the source height. Therefore, an oscillatory ducted mode can exist only in a stable layer, and $N^{2}$ must be large enough for the Scorer parameter to be larger than $k^{2}$.

Another important aspect related to the vertical propagation of the waves is the presence of critical levels
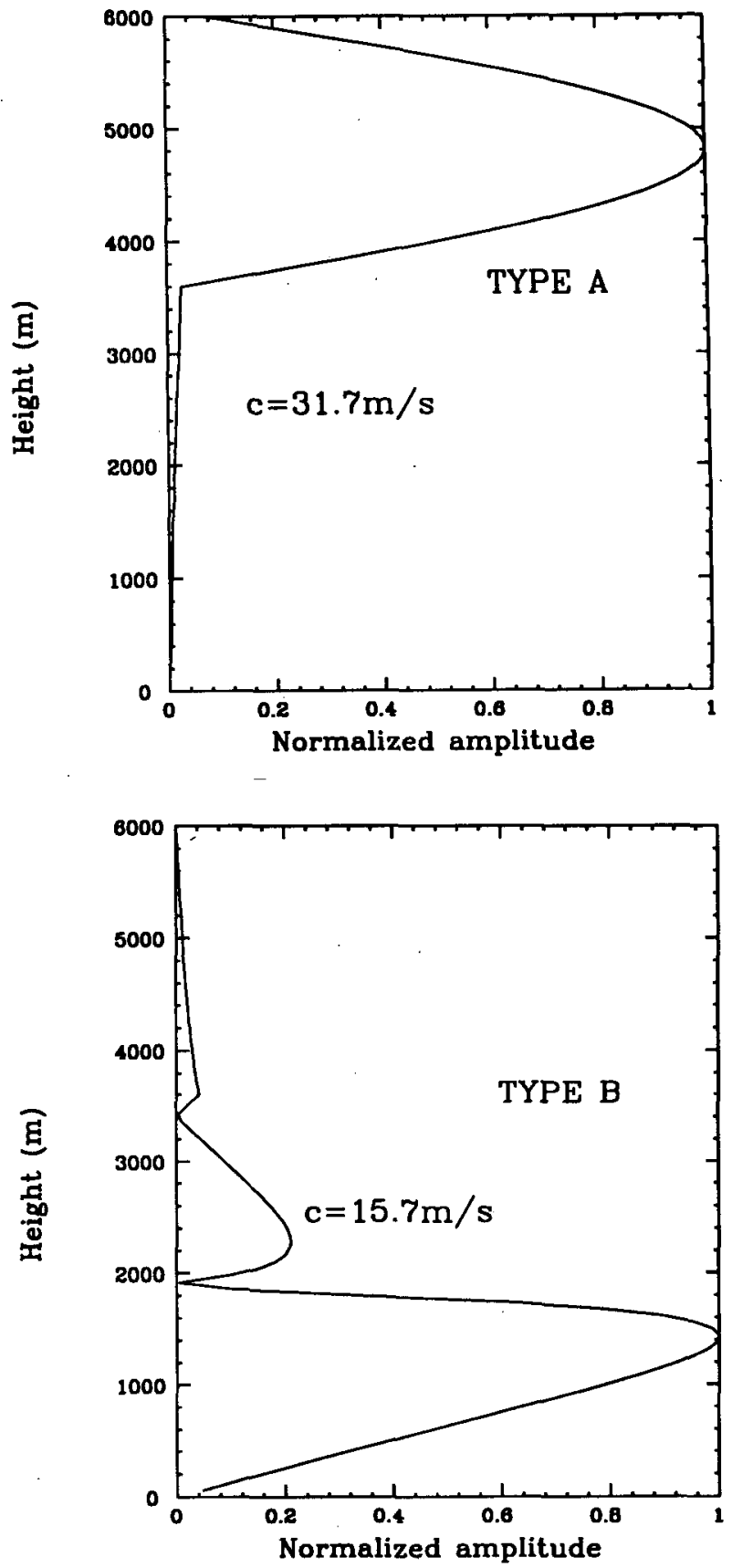

FIG. 5. The modulus of the normalized vertical velocity for the two types of neutral modes that the profiles from Fig. $1 \mathrm{~b}$ can support. The wavenumber is $k=8 \times 10^{-4} \mathrm{~m}^{-1}$.

where the Scorer parameter approaches infinity for neutral modes. The vertical velocity of neutral modes becomes zero at these levels, and the energy is reflected if the layer is unstable or absorbed if it is stable (Booker and Bretherton 1967).

As LT indicate in their paper, to be a ducted mode a wave should not have a critical level in the duct be- 

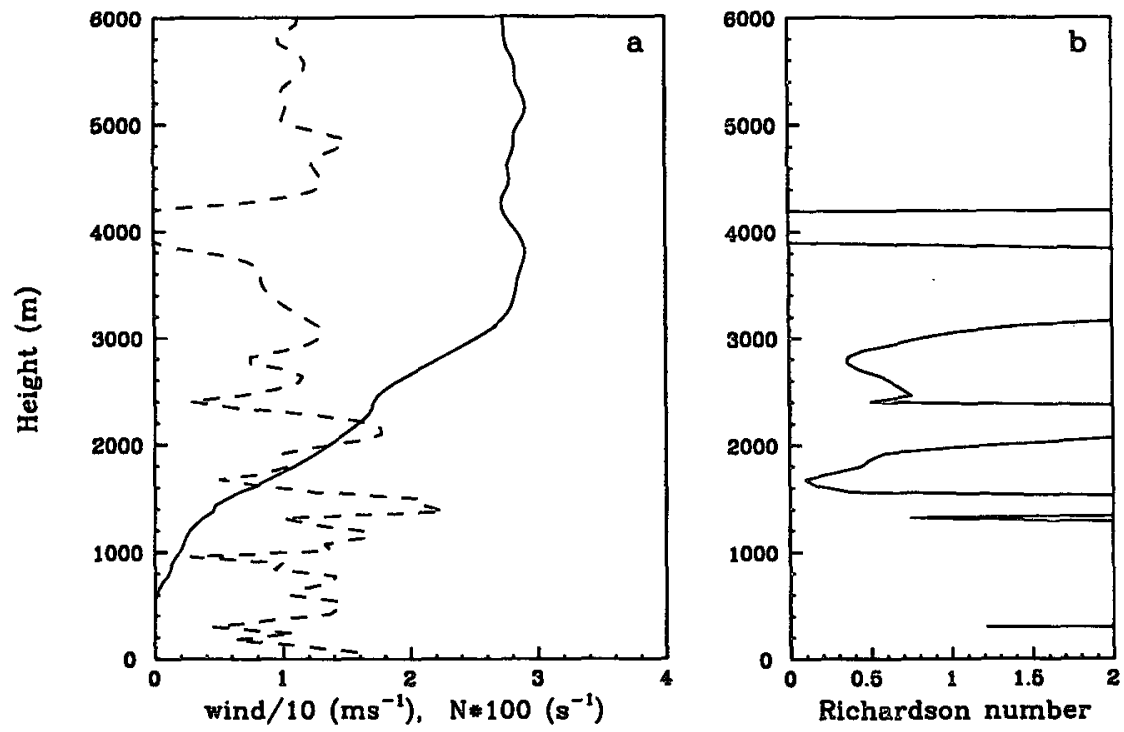

FIG. 6. (a) Wind speed resolved in the measured direction of propagation of the waves $\left(230^{\circ}\right)$ (solid line), Brunt-Väisälä frequency (dashed line), and (b) Richardson number, corresponding to the radiosonde ascent made at Palma de Mallorca at 0000 UTC 25 September 1990.

cause if it did, then wave energy would be absorbed rather than reflected. However, if there is wind shear within the duct, then it is clear that the modes will have a critical level at different heights. This is likely to be important in determining the dominant mode in the duct. The dominant ducted mode was considered by LT to be the one with the largest phase speed because the other ones, with shorter vertical wavelengths, would be more significantly attenuated by any dissipative process. In a duct with constant wind shear, there will be modes with phase speeds equal to the wind at some level inside the duct. These will, following the reasoning of Miles (1961) or Booker and Bretherton (1967), be absorbed at their critical level. This means that in the presence of wind shear the duct has another mechanism aside from dissipation for selecting the dominant trapped mode.

This analysis suggests that wind shear, or any other vertical variation in $\lambda$, plays an important role in determining the expected ducted modes.

We can now summarize these conclusions for the types of neutral waves found numerically.

1) Type A have almost no response near the ground and are confined in some upper layer. The wave energy is expected to remain aloft and so the waves are not going to be observed at the surface.

2) Type B1 are modes of a steplike profile which correspond to singular neutral modes of continuous profiles.

3) Type B2 have a phase speed equal to the wind speed at some level in the intermediate layer, and, using the LT argument, these waves would be trapped between the surface and a critical level situated in a layer where $\mathrm{Ri}<0.25$. The wave energy would be reflected at this level and remain trapped in the layer near the surface. Therefore, this mode is going to be the dominant one at the surface.

4) Type B3 have a phase speed greater than the maximum wind in the domain, so it is expected its energy is not going to be confined in the duct and, therefore, they are not likely to be observed at the ground. This solution is found due to the use of a rigid lid. It is expected that with a radiative upper-boundary condition the wave would radiate away vertically or (more likely) this type of mode would not be found because it depends on a standing wave pattern being set up between the critical level and the rigid upper boundary, which would not be present with a radiative upperboundary condition. Thus, this type of mode can be considered to be spurious and nonphysical.

This means that we expect the dominant modes at the surface, for any wavelength, to be type B2 modes. These will have a phase speed in the range of the wind speed over the intermediate layer. If this range is not large, then the waves will be observed at the surface to be essentially nondispersive.

\section{Use of the method in a case study}

Time series of surface atmospheric pressure from three microbarographs situated on Mallorca (Balearic islands) were used by Monserrat and Thorpe (1992, MT hereafter) to obtain the horizontal properties of some traveling gravity wave events observed in summer 1990. The measured waves propagated with a phase speed of about $29 \mathrm{~m} \mathrm{~s}^{-1}$ and were basically non- 
dispersive. Wavelength, phase speed, and wave direction of propagation for one of the episodes presented by MT are given in Fig. 13 of that paper. Both phase speed and wave direction are basically independent of frequency. In this section, we are going to refer mainly to this episode, the one with largest pressure oscillations, which is referred to as case 3 in MT.

The nondispersive nature of the waves is an indicator suggesting that the waves could be generated by dynamic instability. Waves generated by dynamic instability tend to travel with phase speeds and directions matching the wind speeds and directions at the level of the minimum Richardson number and they are nondispersive. Monserrat and Thorpe found that, although this was not the case in every episode, in case 3 the phase speeds of the waves were very similar to the wind speed at the level of the minimum Richardson number and that the direction of propagation of the wave was just about $20^{\circ}$ different from the wind direction. On the other hand, although in case 3 the Richardson number is actually zero in a layer of about $500 \mathrm{~m}$, in some of the other observed cases the Richardson number is not small enough for dynamic instability. If we assume that just one mechanism is responsible for the generation of every gravity wave episode, we cannot prove at this point that this mechanism is dynamical instability. Whatever the source mechanism, the nondispersive nature of the waves has to be explained. Another point to be considered is that the available soundings are probably more representative of the propagation region of the wave rather than of the generation region, and they are not adequate to find evidence of dynamic instability.

On the other hand, good correlations between two instruments separated by a relatively long distance (more than half the measured horizontal wavelength) were observed. So, whatever the source mechanism is, the good correlations found can be explained only if the wave becomes neutral and the energy remains trapped near the surface.

The vertical profiles of wind and Brunt-Väisälä frequency fulfill the conditions suggested by LT to have an effective duct trapping the waves, but the idealized vertical profiles used by LT in the stable duct cannot be applied to our case. The vertical profiles of BruntVäisälä frequency, wind speed resolved in the measured direction of propagation of the waves $\left(230^{\circ}\right)$, and Richardson number corresponding to the radiosonde ascent made at Palma de Mallorca on 25 September 1990 at 0000 UTC, the closest sounding in time to the observation of the most energetic waves, are shown in Fig. 6. It can be seen that the wind rapidly increases from calm at the surface to a maximum value of about $29 \mathrm{~m} \mathrm{~s}^{-1}$ at a height of about $3800 \mathrm{~m}$, suggesting that assuming a constant wind profile in the stable layer near the ground may introduce spurious results. It is expected then that the presence of wind shear in the duct will introduce some modifications, as discussed in the previous sections.

First, we use the matrix method described in the appendix to compute the unstable modes that the profile shown in Fig. 6 can support. A number of unstable modes with phase speeds ranging between 0 and 29 $\mathrm{m} \mathrm{s}^{-1}$ are found. The most unstable mode has a phase speed of about $6 \mathrm{~m} \mathrm{~s}^{-1}$ and a maximum growth rate of $2.7 \times 10^{-3} \mathrm{~s}^{-1}$ at a wavelength of $450 \mathrm{~m}$. This wavelength is too short to be observed with the array used by MT. For the range of observable wavelengths, say $4-200 \mathrm{~km}$, the maximum growth rate is for $\lambda=4$ $\mathrm{km}$, with a maximum growth rate of $4 \times 10^{-4} \mathrm{~s}^{-1}$, corresponding to an $e$-folding time of $42 \mathrm{~min}$. Consequently, for other observable wavelengths the growth rates are lower. For example, the maximum growth rate for a wavelength of $15 \mathrm{~km}$ is $2 \times 10^{-4} \mathrm{~s}^{-1}$ (e-folding time greater than $1-\mathrm{h})$. We do not really know the timescale of the processes maintaining the unstable profiles in Fig. 6, but it is unlikely that any unstable profile could be maintained for so long. Only with a high frequency of special soundings could the continuous presence of such convective forcing be ascertained. However, we do not expect these unstable modes to have much influence on the surface measurements.

We are interested in knowing if the real atmospheric profile corresponding to this case can support neutral waves with the observed phase speed and if these waves are nondispersive. Therefore, the matrix method described in the appendix is applied to the profile shown in Fig. 6 for different values of the wavenumber $k$, corresponding to wavelengths ranging between 4 and $200 \mathrm{~km}$, to compare the results with the observations.

When the method is applied to real atmospheric profiles, for each wavenumber a number of neutral modes with different phase speeds are obtained. In Fig. 7, the modulus of the vertical velocity for the trapped neutral mode with the largest phase speed is plotted versus height for two different wavelengths, 125 and $4 \mathrm{~km}$. The phase speed of the mode is also indicated in the figure. The mode is clearly confined between the surface and a layer situated at about $4000 \mathrm{~m}$ and, hence, is a type $\mathrm{B} 2$ mode. These modes have almost no response from $4000 \mathrm{~m}$ upward, suggesting they are not depending on the upper rigid-lid condition. However, the upper rigid lid has been moved to a height of 8500 $\mathrm{m}$ to test the upper boundary influence on the modes. We found the mode characteristics in the lower layer are insensitive to the position of the upper lid, giving confidence they are not a consequence of the upperboundary condition used in the model.

The vertical structure for both wavenumbers is somewhat different near the surface, as can be expected by considering the values of the Scorer parameter. Instead of $l^{2}$, the value of $\lambda_{l}=2 \pi(U-c) / N=2 \pi / l$ for $c=29 \mathrm{~m} \mathrm{~s}^{-1}$ is plotted in Fig. 8. For short wavelengths, the quantity $\left(l^{2}-k^{2}\right)$ becomes negative (or $\lambda_{l}>\lambda$ ), 

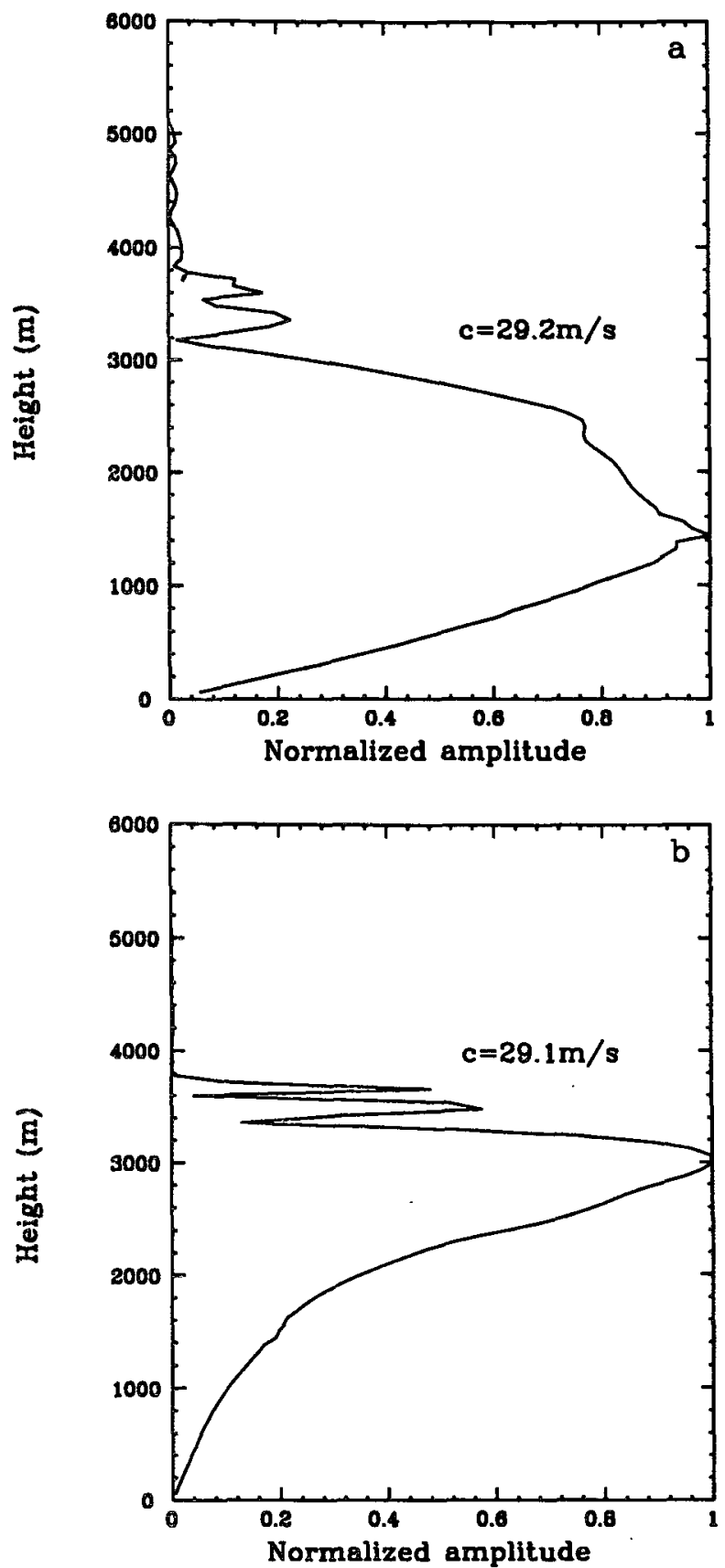

FIG. 7. The modulus of the normalized vertical velocity for the trapped neutral mode with the largest phase speed for (a) $\lambda=125$ $\mathrm{km}$ and (b) $\lambda=4 \mathrm{~km}$. The values of the phase speeds of these modes are indicated.

especially in the lowest kilometers, and the wave is expected to be evanescent in this layer, as is actually found. This can be seen in Fig. 7b, where the wave, with a wavelength of $4 \mathrm{~km}$, has its amplitude maximized at around $3 \mathrm{~km}$ where $\lambda_{l}<\lambda$.

Another aspect that cannot be separated from the propagation of the waves is the height at which they are actually generated. If we assume that the waves are generated by dynamic instability, they would be generated at a height of about $4000 \mathrm{~m}$, where $\mathrm{Ri}<0.25$. It is clear then that very little of the energy would reach the surface if the wave encounters its critical level on its way down. When the phase speed of the mode is smaller than about 28-29 $\mathrm{m} \mathrm{s}^{-1}$ (type B2 modes), this is going to happen at some height between the generation height and the surface, and the wave is not going to be observed at the surface.

Therefore, from the different modes that the actual atmospheric profile can support, we should observe the modes that are trapped by the layer where the Richardson number is less than 0.25 and that, in our case, are also the confined modes with the largest phase speeds. The phase speeds of these modes range between 28 and $29 \mathrm{~m} \mathrm{~s}^{-1}$ and are independent of the wavenumber $k$. (We can observe in Fig. 7 that the phase speeds for the two wavenumbers are similar.) Hence, these modes are clearly nondispersive. The results suggest that we should observe waves with a range of wavelengths and with the waves traveling with phase speeds around 28 $29 \mathrm{~m} \mathrm{~s}^{-1}$, in very good agreement with the actual observations.

We can conclude then that the observed waves in this case are probably trapped modes in a duct. The source mechanism is unclear. It could be associated with dynamic instability of the wind shear as suggested by MT, but this cannot be confirmed without a good knowledge of the vertical structure of the atmosphere upstream.

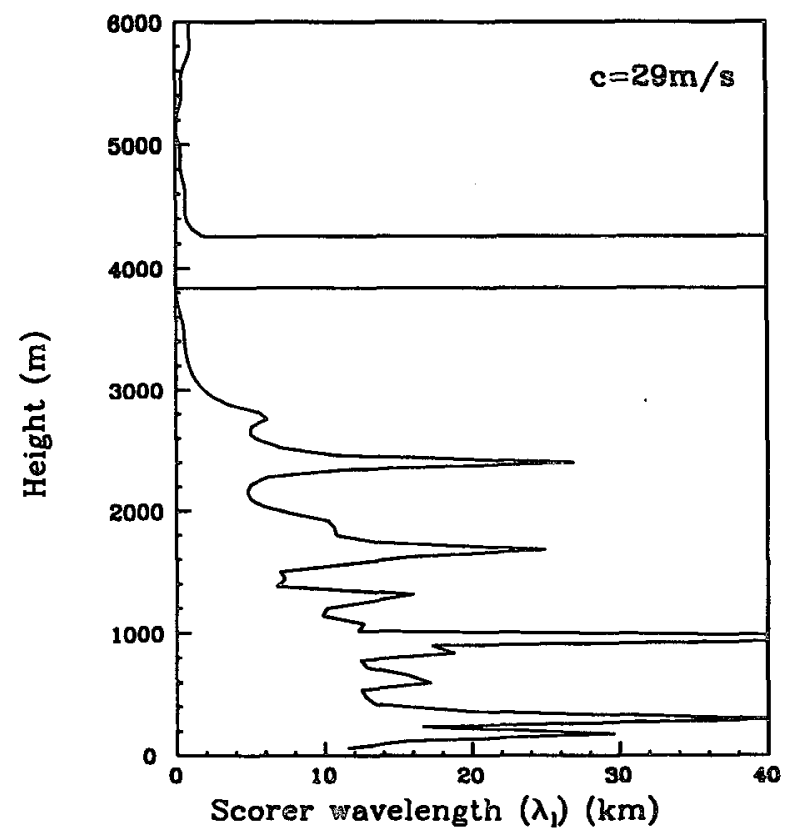

FIG. 8. The wavelength associated with the Scorer parameter $\lambda_{l}$ $=2 \pi(U-c) / N$ for $c=29 \mathrm{~m} \mathrm{~s}^{-1}$, corresponding to the radiosonde ascent made at Palma de Mallorca at 0000 UTC 25 September 1990. 


\section{Summary and conclusions}

The presence of a stable layer capped above by a good reflector is the general condition for the presence of ducted waves in the lower atmosphere. Provided there is an efficient reflecting layer, the ducted wave properties are governed by the characteristics of the stable layer.

For conditions with layers in which the wind speed and the Brunt-Väisälä frequency are constant, a number of free modes can be present in the duct, and their structure can be obtained solving the Taylor-Goldstein equation analytically. Here we show that when wind shear is present in the duct, the number of modes is clearly reduced by wave absorption at critical levels. Thus, shear provides a mechanism by which the spectrum of gravity wave modes in a duct is substantially limited.

In general, when wind and Brunt-Väisälä frequency are functions of height, the gravity wave properties have to be found numerically. A method able to find the neutral modes associated with given atmospheric profiles - the matrix method-has been applied here to the case of constant wind shear in a stable duct. The model results suggest that the dominant mode associated with the vertical atmospheric profiles observed during the gravity wave episode has a phase speed similar to the measured one. Also, the nondispersive nature of the gravity waves that compose this mode is reproduced by the model so that the dominant phase speed is essentially independent of the wavelength.

We consider the interpretation given in this paper of the nondispersive nature of the observed waves to be speculative in the sense that we have not described the dynamics of wave absorption and reflection explicitly. Rather, results from theoretical studies of Booker and Bretherton (1967) and Lindzen and Tung (1976) have been applied to the case studied here. Research is needed to describe these processes in a full numerical model simulation. In such simulations, a source of gravity waves would be imposed at various levels, and the horizontal (and vertical) propagation examined. Then, the hypothesis suggested herein concerning wave trapping and surface pressure fluctuations could be fully tested.

Acknowledgments. Part of this work was done during a one-year visit of one of us (SM) to the Department of Meteorology at the University of Reading, under a grant from the Ministerio de Educación y Ciencia of Spain. Economical support from the Project PB89-0428 of the DGICYT of Spain and the British-Spanish Joint Research Programme HB92-030 and HB93-081 is also acknowledged. Finally, the authors want to express their gratefulness to Dr. A. J. Rosenthal for his thoughtful comments, which improved many parts of the original manuscript.

\section{APPENDIX}

\section{Matrix Method for Solving the Taylor-Goldstein Equation}

Lalas and Einaudi (1976) used a method for obtaining the eigenvalues and eigenfunctions of the TaylorGoldstein equation and presented the results for a hyperbolic tangent wind profile with constant BruntVäisälä frequency. This method, with some modifications, has been used to analyze actual radiosonde ascents (Lalas and Einaudi 1980; Mobbs and Darby 1989). The method, which has been referred to as "the shooting method," is applicable to both neutral and unstable modes and basically consists of solving Eq. (1) by successive iterations, beginning with a first guess for the eigenvalue $c$. The major problem associated with this method is the presence of critical levels where the phase speed of the wave is equal to the wind speed. Some technique is necessary to deal with such levels as some terms in $\lambda(z)$ tend to infinity and Eq. (1) is not applicable. Another problem is the sometimes observed tendency of the method to converge to one particular mode, and, as a result, other modes are never found. If the first guess is far from the real solution, the method may converge very slowly or even diverge. In any case, the necessity of this first guess clearly introduces an unavoidable bias in the final results. Different techniques have been used to minimize these problems, and the method has been successfully used on many occasions, particularly for stability analysis.

Another method for solving the Taylor-Goldstein equation, which has been used much less in the literature, is the so-called matrix method. The method consists of reducing the solution of the Taylor-Goldstein equation to finding the eigenvalues and eigenfunctions of a given matrix. The method is more expensive in computer time, but it has the advantage of not requiring a first guess, avoiding any biased result, and no special technique is necessary to deal with the critical levels. This method is then very useful when seeking neutral modes. The matrix method assumes the atmosphere to be built up from $M$ layers of depth $h$, within which the parameters are considered to be constant. We can then express $U$ and $N^{2}$ as vectors of $M$ components - each component being the value of $U$ and $N^{2}$ in this layer and assumed to be constant throughout each layer. Similarly, the function $W(z)$ is also a vector of $M$ components. On the other hand, the second derivative can be approximated by a simple second order finite difference.

In general, $\lambda(z)$ in Eq. (1) is a complicated function of $c, k, U(z)$, and $N^{2}(z)$. However, to simplify the algebra in describing the method, a simple version of $\lambda$ is first assumed:

$$
\lambda^{2}(z)=\frac{N^{2}}{(U-c)^{2}}-k^{2} .
$$


This is valid when neglecting compressible terms and those involving the second derivative of $U$.

On this basis, Eq. (1) can be rewritten in the form

$$
\begin{aligned}
\left\{c ^ { 2 } \left[\frac{d^{2}}{d z^{2}}-\right.\right. & \left.k^{2}\right]+c\left[-2 U\left(\frac{d^{2}}{d z^{2}}-k^{2}\right)\right] \\
+ & {\left.\left[U^{2}\left(\frac{d^{2}}{d z^{2}}-k^{2}\right)+N^{2}\right]\right\} W=0, }
\end{aligned}
$$

or

$$
\left\{c^{2} \mathbf{A}-c \mathbf{B}-\mathbf{D}\right\} \mathbf{W}=0,
$$

where A, B, and D are matrices of $M$ rows and $M$ columns that, with the boundary conditions $W_{0}=0$ and $W_{M+1}=0$, would have the form

$$
\begin{gathered}
\mathbf{B}=2 \mathbf{U A} \\
\mathbf{D}=-\mathbf{U}^{2} \mathbf{A}-\mathbf{N}^{2} \mathbf{I},
\end{gathered}
$$

where $\mathbf{I}$ is the identity matrix and $\mathbf{A}$ is a tridiagonal matrix:

$$
\mathbf{A}=\left(\frac{d^{2}}{d z^{2}}-k^{2}\right)=\frac{1}{h^{2}}\left(\begin{array}{ccccccc}
-2-k^{2} h^{2} & 1 & 0 & & 0 & 0 & \\
1 & -2-k^{2} h^{2} & 1 & & 0 & 0 & \\
0 & 1 & -2-k^{2} h^{2} & & 1 & 0 & \cdots \\
0 & 0 & 1 & \ddots & & 1 & \\
0 & 0 & 0 & & 1 & & \\
& & \vdots & & & &
\end{array}\right)
$$

Defining now a new vector

$$
\mathbf{W}_{1}=c \mathbf{W},
$$

Eq. (A2) becomes

$$
\mathbf{A}^{-1} \mathbf{D W}+\mathbf{A}^{-1} \mathbf{B} \mathbf{W}_{1}=c \mathbf{W}_{1},
$$

and, using the definition of $W_{1}(A 6)$, and (A7), we can write

$$
\left(\begin{array}{cc}
0 & \mathbf{I} \\
\mathbf{A}^{-1} \mathbf{D} & \mathbf{A}^{-1} \mathbf{B}
\end{array}\right)\left(\begin{array}{c}
\mathbf{W} \\
\mathbf{W}_{1}
\end{array}\right)=c\left(\begin{array}{c}
\mathbf{W} \\
\mathbf{W}_{1}
\end{array}\right)
$$

or

$$
\mathbf{H} \boldsymbol{\psi}=c \boldsymbol{\psi}
$$

Here $\psi$ is a $2 M \times 1$ column vector where the first $M$ values are the components of $\mathrm{W}$ and the second $M$ values are the components of $\mathbf{W}_{1}$. Matrix $\mathbf{H}$ is a matrix with $2 M$ rows and $2 M$ columns including all the information about the vertical structure of the atmosphere.

The solutions of the Taylor-Goldstein equation are the eigenvalues and eigenvectors of this specified matrix $\mathbf{H}$. Equation (A9) can be solved by using any commercial subroutine available. The results shown in this work have been obtained by using two subroutines from the Numerical Algorithms Group library (F02AFF when just eigenvalues are required, and F02AGF when both eigenvalues and eigenvectors need to be computed). The output of the method is a set of $2 M$ eigenvalues and eigenfunctions of the problem. Criteria to select some of these modes are then necessary.

When the fully compressible version of $\lambda(z)$ is used in Eq. (1), the matrix equation (A2) is still obtained, but now A, B, and D have a more complicated form indicated with a prime:

$$
\begin{gathered}
A^{\prime}=\mathbf{A}+\mathbf{V}_{\mathbf{A}} \mathbf{I} \\
\mathbf{B}^{\prime}=2 \mathbf{U A}+\mathbf{V}_{\mathbf{B}} \mathbf{I} \\
\mathbf{D}^{\prime}=-\mathbf{U}^{2} \mathbf{A}+\mathbf{V}_{\mathbf{D}} \mathbf{I},
\end{gathered}
$$

where

$$
\begin{aligned}
\mathbf{V}_{\mathbf{A}}= & \frac{1}{4 \boldsymbol{\rho}_{0}^{2}}\left(\frac{d \boldsymbol{\rho}_{0}}{d z}\right)^{2}-\frac{1}{2 \boldsymbol{\rho}_{0}}\left(\frac{d^{2} \boldsymbol{\rho}_{0}}{d z^{2}}\right)+\frac{\mathbf{N}^{2}}{C_{s}^{2}} \quad(\mathrm{~A} 13) \\
\mathbf{V}_{\mathbf{B}}= & -\left(\frac{d^{2} \mathbf{U}}{d z^{2}}\right)-\frac{\mathbb{U}}{\boldsymbol{\rho}_{0}}\left[\frac{-1}{2 \boldsymbol{\rho}_{0}}\left(\frac{d \boldsymbol{\rho}_{0}}{d z}\right)^{2}+\left(\frac{d^{2} \boldsymbol{\rho}_{0}}{d z^{2}}\right)\right] \\
& -\frac{d \mathbf{U}}{d z}\left[\frac{1}{\boldsymbol{\rho}_{0}}\left(\frac{d \boldsymbol{\rho}_{0}}{d z}\right)+\frac{2 g}{C_{s}^{2}} \mathbf{I}\right]+\frac{2 \mathbf{U} \mathbf{N}^{2}}{C_{s}^{2}}(\mathrm{~A} 14) \\
\mathbf{V}_{\mathbf{D}}= & -\mathbf{N}^{2} \\
& +\mathbf{U}\left(\frac{d^{2} \mathbf{U}}{d z^{2}}\right)-\frac{\mathbb{U}^{2}}{2 \boldsymbol{\rho}_{0}}\left[\frac{1}{2 \boldsymbol{\rho}_{0}}\left(\frac{d \boldsymbol{\rho}_{0}}{d z}\right)^{2}-\left(\frac{d^{2} \boldsymbol{\rho}_{0}}{d z^{2}}\right)\right] \\
& +\mathbf{U} \frac{d \mathbf{U}}{d z}\left[\frac{1}{\rho_{0}}\left(\frac{d \boldsymbol{\rho}_{0}}{d z}\right)+\frac{2 g}{C_{s}^{2}} \mathbf{l}\right]-\frac{\mathbf{U}^{2} \mathbf{N}^{2}}{C_{s}^{2}}
\end{aligned}
$$

Here, $\boldsymbol{\rho}_{0}$ is the basic state density, $C_{s}^{2}$ the speed of sound, and $I$ the unit vector.

When compressible terms and those involving the second derivative of $U$ are neglected, $\mathbf{V}_{\mathbf{A}}, \mathbf{V}_{\mathbf{B}}$, and $\mathbf{V}_{\mathbf{D}}$ have the simple form given previously, that is,

$$
\begin{aligned}
& \mathbb{V}_{A}=0 \\
& \mathbb{V}_{B}=0 \\
& \mathbb{V}_{D}=-\mathbf{N}^{2} .
\end{aligned}
$$




\section{REFERENCES}

Booker, J. R., and F. P. Bretherton, 1967: The critical layer for internal gravity waves in a shear flow. J. Fluid Mech., 27, 513-539.

Einaudi, F., 1980: Gravity waves and the atmospheric boundary layer. Atmospheric Planetary Boundary Layer Physics, A. Longhetto, Ed., Elsevier, 77-93.

Fritts, D. C., 1982: Shear excitation of atmospheric gravity waves. $J$. Atmos. Sci., 39, 1936-1952.

_ 1984: Shear excitation of atmospheric gravity waves. Part II: Nonlinear radiation from a free shear layer. J. Atmos. Sci., 41, 524-537.

Goldstein, S., 1931: On the stability of superposed streams of fluid of different densities. Proc. Roy. Soc. London, Ser. A, 132, 524548 .

Gossard, E. E., and W. H. Hooke, 1975: Waves in the Atmosphere: Atmospheric Infrasound and Gravity Waves. Their Generation and Propagation. Elsevier, $456 \mathrm{pp}$.

Howard, L. N., 1961: Note on a paper of John W. Miles. J. Fluid Mech., 10, 509-512.

Lalas, D. P., and F. Einaudi, 1976: On the characteristics of gravity waves generated by atmospheric shear layers. J. Atmos. Sci., 33, $1248-1259$.

- and - 1980: Tropospheric gravity waves: Their detection and influence on rawinsonde balloon data. Quart. J. Roy. Meteor. Soc., 106, 855-864.
Lindzen, R. S., and A. J. Rosenthal, 1976: On the instability of Helmholtz velocity profiles in stably stratified fluids when a lower boundary is present. J. Geophys. Res., 81, 15611571.

$\ldots$, and K. K. Tung, 1976: Banded convective activity and ducted gravity waves. Mon. Wea. Rev., 104, 1602-1617.

_- E. S. Batten, and J. W. Kim, 1968: Oscillations in atmospheres with tops. Mon. Wea. Rev., 96, 133-140.

Miles, J. W., 1961: On the stability of heterogeneous shear flows. $J$. Fluid. Mech., 10, 496-508.

Mobbs, S. D., and M. S. Darby, 1989: A general method for the linear stability analysis of stratified shear flows. Quart. J. Roy. Meteor. Soc., 115, 915-939.

Monserrat, S., and C. Ramis, 1990: Dynamic stability of a three-layer model with discontinuous profiles of wind and temperature. $J$. Atmos. Sci., 47, 2108-2114.

- , and A. J. Thorpe, 1992: Gravity .wave observations using an array of microbarographs in the Balearic islands. Quart. J. Roy. Meteor. Soc., 118, 259-282.

Rosenthal, A. J., and R. S. Lindzen, 1983: Instabilities in a stratified fluid having one critical level. Part I: Results. J. Atmos. Sci., 40, 509-520.

Taylor, G. I., 1931: Effect of variation of density on the stability of superposed streams of fluid. Proc. Roy. Soc. London, Ser. A, 132, 499-523. 